\title{
WEAK BLD MAPPINGS AND HAUSDORFF MEASURE
}

\author{
PIOTR HAJŁASZ, SOHEIL MALEKZADEH AND SCOTT ZIMMERMAN
}

\begin{abstract}
We prove that if $\Phi: X \rightarrow Y$ a mapping of weak bounded length distortion from a quasiconvex and complete metric space $X$ to any metric space $Y$, then for any Lipschitz mapping $f: \mathbb{R}^{k} \supset E \rightarrow X$ we have that $\mathcal{H}^{k}(f(E))=0$ in $X$ if and only if $\mathcal{H}^{k}(\Phi(f(E)))=0$ in $Y$. This generalizes an earlier result of Hajłasz and Malekzadeh where the target space $Y$ was a Euclidean space $Y=\mathbb{R}^{N}$.
\end{abstract}

\section{To Carlo Sbordone on his 70th birthday}

\section{INTRODUCTION}

A mapping $f: X \rightarrow Y$ between metric spaces is said to have a weak bounded length distortion (weak $\mathrm{BLD}$ ) property if there is a constant $M \geq 1$ such that, for all rectifiable curves $\gamma$ in $X$, the length of $f \circ \gamma$ is comparable to that of $\gamma$ in the following sense:

$$
M^{-1} \ell_{X}(\gamma) \leq \ell_{Y}(f \circ \gamma) \leq M \ell_{X}(\gamma) .
$$

This definition was introduced in [3, 4] and it was motivated by earlier work of Martio and Väisälä [14] and Le Donne [11].

Martio and Väisälä [14] introduced mappings of bounded length distortion (BLD). These are mappings $f: \mathbb{R}^{n} \supset \Omega \rightarrow \mathbb{R}^{n}$ defined on an open set $\Omega$ that are open, discrete, sense preserving and satisfy (1.1) for all curves $\gamma$ in $\Omega$, see also [4]. Subsequently, Le Donne [11] introduced mappings of bounded length distortion (BLD) as mappings between metric spaces that satisfy (1.1) for all curves $\gamma$ in $X$, but without the topological requirements of being open, discrete, or sense preserving.

The class of BLD mappings plays a fundamental role in the contemporary development of geometric analysis and geometric topology, especially in the context of branched coverings of metric spaces. See e.g. [1, 5, 6, 7, 8, 9, 10, 11, 12, 13, 15].

It is important to observe that, in general, the class of weak BLD mappings may be much larger than the class of BLD mappings given by Le Donne. Indeed, the identity mapping id : $\mathbb{H}^{n} \rightarrow \mathbb{R}^{2 n+1}$ from the Heisenberg group $\mathbb{H}^{n}$ into Euclidean space is weak BLD. However, it is not BLD since it maps the $t$-axis, which has Hausdorff dimension two with respect to the Carnot-Carathéodory metric, to the Euclidean $t$-axis which has locally finite length.

2010 Mathematics Subject Classification. 28A75, 30L10, 53C17, 54E40.

Key words and phrases. metric spaces, bounded length distortion, Hausdorff measure, Lipschitz mappings, Sard theorem, Heisenberg group.

P.H. was supported by NSF grant DMS-1500647. 
The aim of this paper is to prove Theorem 1.1. This generalizes Theorem 4.2 from [3] in which the same statement was proven for weak BLD mappings from $X$ into a Euclidean space $\mathbb{R}^{N}$. Our proof will follow a similar argument as in [3]. However, a new proof is required as the co-domain $Y$ is no longer Euclidean but is instead an arbitrary metric space. The main difference between the proofs appears at the end where we apply Lemma 2.1 and estimate the length of the curve $\Phi \circ \Gamma$. The arguments in the proof which are in 3 . will only be sketched, and we refer the reader to [3] for more details.

A metric space $(X, d)$ is said to be quasiconvex if there is a constant $C_{q} \geq 1$ such that, for any $x, y \in X$, there is a rectifiable curve $\gamma:[0,1] \rightarrow X$ connecting $x$ and $y$ (i.e. $\gamma(0)=x$ and $\gamma(1)=y$ ) whose length satisfies $\ell(\gamma) \leq C_{q} d(x, y)$. Such a curve $\gamma$ will be called quasiconvex. Note that, if $(X, d)$ is quasiconvex, then any weak BLD mapping $f: X \rightarrow Y$ is $M C_{q}$-Lipschitz.

Theorem 1.1. Let $\left(X, d_{X}\right)$ be a complete and quasiconvex metric space, and let $\left(Y, d_{Y}\right)$ be any metric space. Let $\Phi: X \rightarrow Y$ be a weak BLD mapping. Then for any $k \in \mathbb{N}$ and any Lipschitz map $f: E \rightarrow X$ defined on a measurable set $E \subset \mathbb{R}^{k}$, the following conditions are equivalent:

(1) $\mathcal{H}^{k}(f(E))=0$ in $X$,

(2) $\mathcal{H}^{k}(\Phi(f(E)))=0$ in $Y$.

If there are no rectifiable curves in $X$, then any mapping $\Phi: X \rightarrow Y$ is weak BLD. Thus the assumption that the space $X$ is quasiconvex is a very natural one. Clearly, biLipschitz mappings preserve sets of Hausdorff measure zero, but the weak BLD condition is much weaker than bi-Lipschitz continuity. Recall, the identity map from the Heisenberg group $\mathbb{H}^{n}$ to $\mathbb{R}^{2 n+1}$ is weak BLD. This together with Theorem 1.1] can be used to prove unrectifiabilty of the Heisenberg group (see [3]).

Another application of the theorem is to a result of Gromov. In [2, Theorem 2.4.11], Gromov proved that any Riemannian manifold of dimension $n$ admits a mapping into $\mathbb{R}^{n}$ that preserves lengths of curves. It follows from Theorem 1.1 that the Jacobian of such a mapping is different than zero almost everywhere, and hence there is no curve-length preserving mapping into $\mathbb{R}^{m}$ for $m<n$. While this result is known, Theorem 1.1 provides a new perspective. For other comments and applications see [3] and [4].

We will prove Theorem 1.1 as a consequence of the following result.

Theorem 1.2. Suppose that $(X, d)$ is a complete and quasiconvex metric space, and let $\Phi: X \rightarrow \ell^{\infty}$ be a weak BLD mapping. Then, for any $k \in \mathbb{N}$ and any Lipschitz map $f: E \rightarrow X$ defined on a measurable set $E \subset \mathbb{R}^{k}$, the following conditions are equivalent:

(1) $\mathcal{H}^{k}(f(E))=0$ in $X$,

(2) $\mathcal{H}^{k}(\Phi(f(E)))=0$ in $\ell^{\infty}$,

(3) $\operatorname{rank}(\operatorname{ap} D(\Phi \circ f))<k$ a.e. in $E$.

The last condition (3) requires some explanation. Let $g=\left(g_{1}, g_{2}, \ldots\right): \mathbb{R}^{k} \supset E \rightarrow \ell^{\infty}$ be an $L$-Lipschitz mapping. Then the components $g_{i}: E \rightarrow \mathbb{R}$ are also $L$-Lipschitz. Hence, for $\mathcal{H}^{k}$-almost all points $x \in E$, the functions $g_{i}, i \in \mathbb{N}$ are approximately differentiable at 
$x \in E$. We define the approximate derivative of $g$ component-wise as follows:

$$
\operatorname{ap} D g(x)=\left[\begin{array}{c}
\text { ap } D g_{1}(x) \\
\operatorname{ap} D g_{2}(x) \\
\vdots
\end{array}\right\rceil
$$

For each $i \in \mathbb{N}$, ap $D g_{i}(x)$ is a vector in $\mathbb{R}^{k}$ with components bounded by $L$. Thus ap $D g(x)$ can be regarded as an $\infty \times k$ matrix of real numbers whose components are bounded by $L$. It is easy to see that, for an $\infty \times k$ matrix, the row rank equals the column rank. Indeed, the rank-nullity theorem still holds for such matrices. Therefore, the rank of ap $D g(x)$ is always at most $k$.

The paper is structured as follows. In Section 2 we show how to deduce Theorem 1.1 from Theorem 1.2, and in Section 3 we prove Theorem 1.2 .

\section{Proof of Theorem 1.1 from Theorem 1.2}

If $Y$ is a separable space, Theorem 1.1 follows very easilly from Theorem 1.2. Indeed, every separable metric space admits an isometric (Kuratowski) embedding $\kappa: Y \rightarrow \ell^{\infty}$, and the composition $\kappa \circ \Phi: X \rightarrow \ell^{\infty}$ is still a weak BLD mapping. Thus for any Lipschitz mapping $f: \mathbb{R}^{k} \supset E \rightarrow X$, Theorem 1.2 implies that $\mathcal{H}^{k}(f(E))=0$ in $X$ if and only if $\mathcal{H}^{k}((\kappa \circ \Phi)(f(E)))=0$ in $\ell^{\infty}$. However, the last condition is equivalent to $\mathcal{H}^{k}(\Phi(f(E)))=0$ since isometries preserve Hausdorff measure and hence

$$
\mathcal{H}^{k}(\Phi(f(E)))=\mathcal{H}^{k}(\kappa(\Phi(f(E)))) .
$$

If $Y$ is not separable, the arguments are slightly more complicated. The metric space $\Phi(f(E)) \subset Y$ with the induced metric is separable, so it admits an isometric embedding $\kappa: \Phi(f(E)) \rightarrow \ell^{\infty}$. We would like to mimic the above argument, but there is a technical issue: the mapping $\kappa \circ \Phi$ is defined only on the set $f(E) \subset X$, and in general this set is neither quasiconvex nor complete as a metric space with the induced metric. It is, however, a separable subset of $X$. We may thus use the following lemma to reduce $X$ to a separable, quasiconvex, complete space $\tilde{X}$ containing $f(E)$.

Lemma 2.1. Let $(X, d)$ be a quasiconvex and complete metric space and let $A \subset X$ be a separable subset. Then there is a subset $\tilde{X} \subset X$ containing $A$ such that $(\tilde{X}, d)$ is separable, quasiconvex, and complete.

Before proving the lemma, we will show how to use it to complete the proof of Theorem 1.1. Set $A=f(E)$ and choose the space $\tilde{X}$ as in the lemma. Since $\tilde{X}$ is separable, so too is $\Phi(\tilde{X}) \subset Y$. We thus have an isometric embedding $\tilde{\kappa}: \Phi(\tilde{X}) \rightarrow \ell^{\infty}$, and so the mapping $\tilde{\kappa} \circ \Phi: \tilde{X} \rightarrow \ell^{\infty}$ is weak BLD. Thus it follows from Theorem 1.2 that $\mathcal{H}^{k}(f(E))=0$ in $\tilde{X}$ (and thus in $X$ ) if and only if

$$
\mathcal{H}^{k}(\Phi(f(E)))=\mathcal{H}^{k}(\tilde{\kappa}(\Phi(f(E))))=0
$$

in $Y$. This completes the proof of Theorem 1.1. It remains to prove the lemma. 
Proof of Lemma 2.1. Choose a countable and dense subset $A_{1}=\left\{x_{i}\right\}_{i=1}^{\infty}$ of $A$. For any $i, j \in \mathbb{N}$, choose a quasiconvex curve $\gamma_{i j}:[0,1] \rightarrow X$ connecting $x_{i}$ to $x_{j}$. Define the set $\mathcal{J}_{1}:=\left\{\gamma_{i j}\right\}_{i, j=1}^{\infty}$. This is a countable family of quasiconvex curves connecting all pairs of points in $A_{1}$.

Suppose by way of induction that the countable set $A_{n}$ and countable family of curves $\mathcal{J}_{n}$ have been defined. Define $A_{n+1}$ to be a countable, dense subset of $\bigcup_{\gamma \in \mathcal{J}_{n}} \gamma([0,1])$ such that $A_{n+1} \supset A_{n}$. This is possible since each $\gamma([0,1])$ is separable and since the family $\mathcal{J}_{n}$ is countable. As above, define $\mathcal{J}_{n+1}$ to be a countable family of quasiconvex curves connecting all pairs of points in $A_{n+1}$ by selecting one curve for each pair of points.

Set $\tilde{A}=\bigcup_{n=1}^{\infty} A_{n}$, and define $\tilde{X}$ to be the closure of $\tilde{A}$. Clearly, $A \subset \tilde{X}$, and $\tilde{X}$ is separable. Moreover, $\tilde{X}$ is complete as it is a closed subset of a complete space. It remains to show that $(\tilde{X}, d)$ is quasiconvex. If $x, y \in \tilde{A}$, then $x, y \in A_{n}$ for some $n$ (because $\left.A_{1} \subset A_{2} \subset A_{3} \subset \ldots\right)$. Hence the points $x$ and $y$ can be connected by a quasiconvex curve $\gamma$ that belongs to $\mathcal{J}_{n}$. Since $A_{n+1} \cap \gamma([0,1])$ is dense in $\gamma([0,1])$, we have that $\gamma([0,1]) \subset \tilde{X}$.

Let $\varepsilon>0$. Fix $x, y \in \tilde{X}$ with $x \neq y$. Then there are sequences $\left\{x_{k}\right\}_{k=1}^{\infty}$ and $\left\{y_{k}\right\}_{k=1}^{\infty}$ in $\tilde{A}$ with

$$
d\left(x_{k}, x\right) \leq \varepsilon d(x, y) 2^{-k} \quad \text { and } \quad d\left(y_{k}, y\right) \leq \varepsilon d(x, y) 2^{-k} .
$$

It easily follows from the triangle inequality that

$$
d\left(x_{k}, x_{k+1}\right) \leq \varepsilon d(x, y) 2^{-(k-1)} \quad \text { and } \quad d\left(y_{k}, y_{k+1}\right) \leq \varepsilon d(x, y) 2^{-(k-1)}
$$

Also

$$
d\left(x_{1}, y_{1}\right) \leq(1+\varepsilon) d(x, y)
$$

Hence

$$
d\left(x_{1}, y_{1}\right)+\sum_{k=1}^{\infty} d\left(x_{k}, x_{k+1}\right)+\sum_{k=1}^{\infty} d\left(y_{k}, y_{k+1}\right) \leq(1+5 \varepsilon) d(x, y) .
$$

By the arguments above, we may connect $x_{1}$ and $y_{1}$ by a quasiconvex curve $\gamma_{0}$ in $\tilde{X}$ of length at most $C_{q} d\left(x_{1}, y_{1}\right)$. Here, $C_{q}$ is the quasiconvexity constant associated with $X$. For $k \in \mathbb{N}$ we connect $x_{k}$ to $x_{k+1}$ by a quasiconvex curve $\alpha_{k}$ in $\tilde{X}$ of length at most $C_{q} d\left(x_{k}, x_{k+1}\right)$ and connect $y_{k}$ to $y_{k+1}$ by a quasiconvex curve $\beta_{k}$ in $\tilde{X}$ of length at most $C_{q} d\left(y_{k}, y_{k+1}\right)$. Concatenating these curves in the obvious order creates a rectifiable curve $\gamma$ in $\tilde{X}$ with length

$$
\ell(\gamma)=\ell\left(\gamma_{0}\right)+\sum_{k=1}^{\infty} \ell\left(\alpha_{k}\right)+\sum_{k=1}^{\infty} \ell\left(\beta_{k}\right) \leq C_{q}(1+5 \varepsilon) d(x, y) .
$$

The curve $\gamma$ connects $x$ and $y$. Therefore, the space $\tilde{X}$ is quasiconvex with any quasiconvexity constant larger than $C_{q}$.

\section{Proof of Theorem 1.2}

Suppose that $(X, d)$ is complete and quasiconvex and $\Phi: X \rightarrow \ell^{\infty}$ is weak BLD. Suppose also that $E \subset \mathbb{R}^{k}$ and $f: E \rightarrow X$ is Lipschitz. The implication from (1) to (2) is obvious 
because the mapping $\Phi$ is Lipschitz. The equivalence between (2) and (3) follows from the following result [3, Theorem 2.2]:

Lemma 3.1. Let $E \subset \mathbb{R}^{k}$ be measurable and let $g: E \rightarrow \ell^{\infty}$ be a Lipschitz mapping. Then $\mathcal{H}^{k}(g(E))=0$ if and only if $\operatorname{rank}(\operatorname{ap} D g(x))<k, \mathcal{H}^{k}$-a.e. in $E$.

The implication from left to right is easy: $g$ composed with a projection $\pi$ of $\ell^{\infty}$ onto any $k$-dimensional subspace generated by a choice of $k$-coordinates in $\ell^{\infty}$ is Lipschitz, so $\mathcal{H}^{k}(\pi(g(E)))=0$. Hence, by the area formula, the determinant of the mapping $\pi \circ g$ equals zero a.e. This implies that $\operatorname{rank}(\operatorname{ap} D g(x))<k$ a.e.

The reverse implication is much more difficult and follows the Sard type arguments seen in the remainder of this paper. For details, see [3].

It remains to prove that (3) implies (1). Suppose that $\operatorname{rank}(\operatorname{ap} D(\Phi \circ f))<k$ a.e. in $E$. Let $\varepsilon>0$. Since $(\Phi \circ f)_{i}: E \rightarrow \mathbb{R}$ is Lipschitz for each $i \in \mathbb{N}$, we can find $g_{i} \in C^{1}\left(\mathbb{R}^{k}\right)$ with

$$
\mathcal{H}^{k}\left(\left\{x \in E:(\Phi \circ f)_{i}(x) \neq g_{i}(x)\right\}\right)<\varepsilon / 2^{i}
$$

and ap $D\left((\Phi \circ f)_{i}\right)(x)=D g_{i}(x)$ for almost every $x \in E$ at which $(\Phi \circ f)_{i}(x)=g_{i}(x)$. Thus there is a measurable set $F \subset E$ such that $\mathcal{H}^{k}(E \backslash F)<\varepsilon$ and

$$
\Phi \circ f=\left(g_{1}, g_{2}, \ldots\right)=: g, \quad \text { ap } D(\Phi \circ f)=\left[\begin{array}{c}
D g_{1} \\
D g_{2} \\
\vdots
\end{array}\right]=: D g
$$

at all points of $F$. Let

$$
\tilde{F}=\{x \in F: \operatorname{rank} \text { ap } D(\Phi \circ f)(x)=\operatorname{rank} D g(x)<k\} .
$$

By assumption, $\mathcal{H}^{k}(F \backslash \tilde{F})=0$. Recall that our goal is to prove $\mathcal{H}^{k}(f(E))=0$. It suffices to prove $\mathcal{H}^{k}(f(\tilde{F}))=0$ since we may exhaust $E$ by sets $\tilde{F}$ up to a set of $\mathcal{H}^{k}$-measure zero and since $f$ maps sets of $\mathcal{H}^{k}$-measure zero to sets of $\mathcal{H}^{k}$-measure zero. Moreover, the set $\tilde{F}$ can be decomposed as follows:

$$
\tilde{F}=\bigcup_{j=0}^{k-1} K_{j}, \quad K_{j}=\{x \in \tilde{F}: \operatorname{rank} D g(x)=j\} .
$$

Thus it suffices to show that $\mathcal{H}^{k}\left(f\left(K_{j}\right)\right)=0$ for $j=0,1,2, \ldots, k-1$. By removing a set of measure zero we can assume that all points of $K_{j}$ are density points of $K_{j}$.

In fact, it suffices to prove that any point in $K_{j}$ has a cubic neighborhood whose intersection with $K_{j}$ is mapped onto a set of $\mathcal{H}^{k}$-measure zero. In the remainder of the paper, a "cube" will refer to a cube with edges parallel to the coordinate axes.

For each $j \geq 1$, we may apply the change of variables [3, Lemma 2.6] as in the proof of [3. Theorem 2.2] and assume that

$$
K_{j} \subset(0,1)^{k} \text { and } g_{i}(x)=x_{i} \text { for } i=1,2, \ldots, j \text { and } x \in[0,1]^{k} .
$$

Since rank $D g(x)=j$ for any $x \in K_{j}$ and since $g$ fixes the first $j$ coordinates of $x$, we have

$$
\frac{\partial g_{\ell}}{\partial x_{i}}(x)=0 \quad \text { for } x \in K_{j}, i=j+1, \ldots, k \text { and all } \ell=1,2, \ldots
$$


If $j=0$ we do not need to apply a change of variables.

Now the result will follow from the next lemma after a standard application of the Vitali type $5 r$-covering lemma. Indeed, it allows us to cover $K_{j}$ by cubes $Q_{x_{i}}$ so that the cubes $5^{-1} Q_{x_{i}}$ are pairwise disjoint and thus bound the Hausdorff content $\mathcal{H}_{\infty}^{k}\left(f\left(K_{j}\right)\right)$ by $C m^{j-k}$ for some $C>0$, and this can be made arbitrarily small since $m$ is arbitrary and $j-k<0$. See the argument following the statement of Lemma 2.7 in [3] for full details.

Lemma 3.2. Let $M$ be the BLD constant of $\Phi$, let $C_{q}$ be the quasiconvexity constant of $X$, and let $L$ be the Lipschitz constant of $f$. Under the assumptions (3.1), there is a constant $C=C(k) C_{q}^{2} M^{2}>0$ such that, for any integer $m \geq 1$ and $x \in K_{j}$, there is a closed cube $Q_{x} \subseteq[0,1]^{k}$ centered at $x$ of edge length $d_{x}$ such that $f\left(K_{j} \cap Q_{x}\right)$ can be covered by $m^{j}$ balls in $X$, each of radius $C L d_{x} m^{-1}$.

Proof. Since $x$ is a density point of $K_{j}$, there is a cube $Q \subseteq[0,1]^{k}$ centered at $x$ with edge length $d$ such that $\mathcal{H}^{k}\left(Q \backslash K_{j}\right)<m^{-k} d^{k}$. We can assume that $Q=[0, d]^{k}$. Divide $[0, d]^{j}$ into cubes $\left\{Q_{\nu}\right\}_{\nu=1}^{m^{j}}$ of edge length $d / m$ with pairwise disjoint interiors. We want to prove that, inside each rectangular box $Q_{\nu} \times[0, d]^{k-j}$, the set $K_{j}$ is mapped by $f$ into a small ball. In particular, we want

$$
\operatorname{diam}_{X}\left(f\left(\left(Q_{\nu} \times[0, d]^{k-j}\right) \cap K_{j}\right)\right)<C(k) C_{q}^{2} M^{2} L d m^{-1} .
$$

Since $\mathcal{H}^{k}\left(\left(Q_{\nu} \times[0, d]^{k-j}\right) \backslash K_{j}\right) \leq \mathcal{H}^{k}\left(Q \backslash K_{j}\right)<m^{-k} d^{k}$, we may use Fubini's theorem as in the proof of [3. Lemma 2.7] to find $\rho \in Q_{\nu}$ such that

$$
\mathcal{H}^{k-j}\left(\left(\{\rho\} \times[0, d]^{k-j}\right) \backslash K_{j}\right)<m^{j-k} d^{k-j} .
$$

In particular, every point in $\{\rho\} \times[0, d]^{k-j}$ is at a distance no more than $C(k) m^{-1} d$ from the set $\left(\{\rho\} \times[0, d]^{k-j}\right) \cap K_{j}$. Hence every point in $Q_{\nu} \times[0, d]^{k-j}$ (and thus every point in $\left.\left(Q_{\nu} \times[0, d]^{k-j}\right) \cap K_{j}\right)$ is at a distance at most $C(k) m^{-1} d$ from the set $\left(\{\rho\} \times[0, d]^{k-j}\right) \cap K_{j}$. Since $f$ is $L$-Lipschitz, in order to prove (3.3) it suffices to show that

$$
\operatorname{diam}_{X}\left(f\left(\left(\{\rho\} \times[0, d]^{k-j}\right) \cap K_{j}\right)\right)<C(k) C_{q}^{2} M^{2} L d m^{-1} .
$$

To begin to prove (3.5), we will recall Lemma 4.4 from [3].

Lemma 3.3. Let $E \subset \mathcal{Q}$ be a measurable subset of a cube $\mathcal{Q} \subset \mathbb{R}^{n}$. Then there is a constant $C=C(n)>0$ such that, for any $x \in \mathcal{Q}$,

$$
\mathcal{H}^{n}\left(\left\{y \in \mathcal{Q}: \mathcal{H}^{1}(\overline{x y} \cap E) \leq C \mathcal{H}^{n}(E)^{1 / n}\right\}\right)>\frac{\mathcal{H}^{n}(\mathcal{Q})}{2}
$$

where $\overline{x y}$ is the segment from $x$ to $y$.

This lemma implies that, if the measure of $E \subset \mathcal{Q}$ is small, then more than half of the intervals in $\mathcal{Q}$ intersect $E$ along a short subset. See [3, Lemma 4.4] for a short proof.

Under the assumptions of Lemma 3.3, for any $x, y \in \mathcal{Q}$ we can find $z \in \mathcal{Q}$ such that

$$
\mathcal{H}^{1}(\overline{x z} \cap E)+\mathcal{H}^{1}(\overline{z y} \cap E) \leq C \mathcal{H}^{n}(E)^{1 / n} .
$$

That is, the curve $\overline{x z}+\overline{z y}$ connecting $x$ to $y$ intersects the set $E$ along a subset of length at most $C \mathcal{H}^{n}(E)^{1 / n}$. Notice also that this curve has length no larger than $2 \operatorname{diam}(\mathcal{Q})$. 
Applying this argument with dimension $n=k-j$, cube $\mathcal{Q}=\{\rho\} \times[0, d]^{k-j}$, and subset $E=\left(\{\rho\} \times[0, d]^{k-j}\right) \backslash K_{j}$, every pair of points $x, y \in\left(\{\rho\} \times[0, d]^{k-j}\right) \cap K_{j}$ can be connected by a curve of length at most $2 d \sqrt{k-j}$ (which is two times the diameter of $\{\rho\} \times[0, d]^{k-j}$ ) whose intersection with $\left(\{\rho\} \times[0, d]^{k-j}\right) \backslash K_{j}$ has length no more than $C(k) m^{-1} d$ (by (3.4) $)$.

Fix $x, y \in\left(\{\rho\} \times[0, d]^{k-j}\right) \cap K_{j}$ and choose $\gamma$ to be a curve in $\{\rho\} \times[0, d]^{k-j}$ as described in the previous paragraph. Parametrize $\gamma$ by arc-length so that it is a 1-Lipschitz curve. The mapping $f \circ \gamma$ is $L$-Lipschitz and is defined on the set $\gamma^{-1}\left(K_{j}\right) \subset[0, \ell(\gamma)]$. This map uniquely extends to an $L$-Lipschiz map defined on the closure of $\gamma^{-1}\left(K_{j}\right)$ (since it is Lipschitz and $X$ is complete). The complement of $\overline{\gamma^{-1}\left(K_{j}\right)}$ consists of countably many (relatively) open intervals whose total length is bounded by $C(k) m^{-1} d$. Since the space $X$ is quasiconvex, we can extend $f \circ \gamma$ from $\overline{\gamma^{-1}\left(K_{j}\right)}$ to a $C_{q} L$-Lipschitz curve $\Gamma:[0, \ell(\gamma)] \rightarrow X$ connecting $f(x)$ to $f(y)$. Indeed, we may construct this extension by choosing for each open interval in the complement of $\overline{\gamma^{-1}\left(K_{j}\right)}$ a quasiconvex curve in $X$ (which is $C_{q} L$-Lipschitz on the interval after possibly reparameterizing) that connects the images of the endpoints of the interval.

According to the paragraph preceding the statement of Theorem 1.1, the mapping $\Phi$ is $M C_{q}$-Lipschitz so the curve $\Phi \circ \Gamma:[0, \ell(\gamma)] \rightarrow \ell^{\infty}$ is $M C_{q}^{2} L$-Lipschitz. In order to prove (3.5), it suffices to show that

$$
\ell(\Phi \circ \Gamma) \leq C(k) M C_{q}^{2} L m^{-1} d .
$$

Indeed, since $\Phi$ is weak BLD we would have

$$
d(f(x), f(y)) \leq \ell(\Gamma) \leq M \ell(\Phi \circ \Gamma) \leq C(k) C_{q}^{2} M^{2} L m^{-1} d .
$$

Since we may find such a curve $\Gamma$ for any $x, y \in\left(\{\rho\} \times[0, d]^{k-j}\right) \cap K_{j}$, (3.5) follows.

Thus it remains to prove the estimate (3.7). Since $\Phi \circ \Gamma$ is a curve in $\ell^{\infty}$, the proof of this estimate is slightly more subtle than that of the corresponding estimate in the proof of [3, Theorem 4.2] where the curve was in $\mathbb{R}^{N}$. The proof is a result of the following lemma:

Lemma 3.4. If $\eta=\left(\eta_{1}, \eta_{2}, \ldots\right):[a, b] \rightarrow \ell^{\infty}$ is a Lipschitz curve, then

$$
\ell(\eta) \leq \int_{a}^{b}\left\|\eta^{\prime}(t)\right\|_{\infty} d t, \quad \text { where } \eta^{\prime}=\left(\eta_{1}^{\prime}, \eta_{2}^{\prime}, \ldots\right) .
$$

Proof. For $[s, t] \subset[a, b]$,

$$
\|\eta(t)-\eta(s)\|_{\infty}=\sup _{i}\left|\eta_{i}(t)-\eta_{i}(s)\right| \leq \sup _{i} \int_{s}^{t}\left|\eta_{i}^{\prime}(\tau)\right| d \tau \leq \int_{s}^{t}\left\|\eta^{\prime}(\tau)\right\|_{\infty} d \tau,
$$

so for any partition $a=t_{0}<t_{1}<\cdots<t_{n}=b$ of $[a, b]$, we have

$$
\sum_{k=0}^{n-1}\left\|\eta\left(t_{k+1}\right)-\eta\left(t_{k}\right)\right\|_{\infty} \leq \sum_{k=0}^{n-1} \int_{t_{k}}^{t_{k+1}}\left\|\eta^{\prime}(\tau)\right\|_{\infty} d \tau=\int_{a}^{b}\left\|\eta^{\prime}(\tau)\right\|_{\infty} d \tau
$$

Since $\ell(\eta)$ equals the supremum of the sums on the left hand side over all partitions of $[a, b]$, the lemma follows. 
Note that, on the set $\gamma^{-1}\left(K_{j}\right)$, the curve $\Phi \circ \Gamma$ coincides with $g \circ \gamma$. Thus for almost every $t \in \gamma^{-1}\left(K_{j}\right)$ we have

$$
(\Phi \circ \Gamma)^{\prime}(t)=(g \circ \gamma)^{\prime}(t)=0 .
$$

This is an easy consequence of (3.2) since $\gamma$ is a curve in $\{\rho\} \times[0, d]^{k-j}$. Hence the length of the curve $\Phi \circ \Gamma$ satisfies

$$
\begin{aligned}
\ell(\Phi \circ \Gamma) & \leq \int_{0}^{\ell(\gamma)}\left\|(\Phi \circ \Gamma)^{\prime}(t)\right\|_{\infty} d t \\
& \leq\left(M C_{q}^{2} L\right) \mathcal{H}^{1}\left([0, \ell(\gamma)] \backslash \gamma^{-1}\left(K_{j}\right)\right) \\
& \leq C(k) M C_{q}^{2} L m^{-1} d
\end{aligned}
$$

which proves (3.7). The proof is complete.

\section{REFERENCES}

[1] Drasin, D., Pankka, P.: Sharpness of Rickman's Picard theorem in all dimensions. Acta Math. 214, no. 2 (2015), 209-306.

[2] Gromov, M.: Partial differential relations. Ergebnisse der Mathematik und ihrer Grenzgebiete (3) [Results in Mathematics and Related Areas (3)], 9. Springer-Verlag, Berlin, 1986.

[3] HajŁasz, P., Malekzadeh, S.: On conditions for unrectifiability of a metric space. Anal. Geom. Metr. Spaces 3 (2015), 1-14.

[4] HajŁasz, P., Malekzadeh, S.: A new characterization of the mappings of bounded length distortion. Int. Math. Res. Not. IMRN 2015, no. 24, 13238-13244.

[5] Heinonen, J., Keith, S.: Flat forms, bi-Lipschitz parameterizations, and smoothability of manifolds. Publ. Math. Inst. Hautes Études Sci. No. 113 (2011), 1-37.

[6] Heinonen, J.; Kilpeläinen, T.: BLD-mappings in $W^{2,2}$ are locally invertible. Math. Ann. 318 (2000), 391-396.

[7] Heinonen, J.; Kilpeläinen, T.; Martio, O.: Harmonic morphisms in nonlinear potential theory. Nagoya Math. J. 125 (1992), 115-140.

[8] Heinonen, J., Rickman, S.: Geometric branched covers between generalized manifolds. Duke Math. J. 113 (2002), 465-529.

[9] Heinonen, J., Rickman, S.: Quasiregular maps $S^{3} \rightarrow S^{3}$ with wild branch sets. Topology 37 (1998), 1-24.

[10] Heinonen, J., Sullivan, D.: On the locally branched Euclidean metric gauge. Duke Math. J. 114 (2002), 15-41.

[11] Le Donne, E.: Lipschitz and path isometric embeddings of metric spaces. Geom. Dedicata 166 (2013), 47-66.

[12] Le Donne, E., Pankka, P.: Closed BLD-elliptic manifolds have virtually Abelian fundamental groups. New York J. Math. 20 (2014), 209-216.

[13] Luisto, R.: A Characterization of BLD-Mappings Between Metric Spaces. J. Geom. Anal. DOI $10.1007 / \mathrm{s} 12220-016-9752-5$

[14] Martio, O., VÄıs̈̈LÄ, J.: Elliptic equations and maps of bounded length distortion. Math. Ann. 282 (1988), 423-443.

[15] PAnkKA, P.: Slow quasiregular mappings and universal coverings. Duke Math. J. 141 (2008), 293320.

P. HajŁasz: Department of Mathematics, University of Pittsburgh, 301 Thackeray Hall, Pittsburgh, PA 15260, USA, hajlasz@pitt.edu

S. Malekzadeh: Department of Mathematics, University of Pittsburgh, 301 Thackeray Hall, Pittsburgh, PA 15260, USA, som13@pitt.edu 
S. Zimmerman: Department of Mathematics, University of Pittsburgh, 301 Thackeray Hall, PittsBurgh, PA 15260, USA, srz5@pitt.edu 